\title{
Further Development of Synchronous Array Method for Ad Hoc Wireless Networks
}

\author{
Yuan Yu, ${ }^{1}$ Yi Huang, ${ }^{1}$ Bin Zhao, ${ }^{2}$ and Yingbo Hua ${ }^{1}$ \\ ${ }^{1}$ Department of Electrical Engineering, University of California, Riverside, CA 92521, USA \\ ${ }^{2}$ Cinea, Inc., 16 S. 17th Street, Richmond, VA 23219, USA
}

Correspondence should be addressed to Yingbo Hua, yhua@ee.ucr.edu

Received 1 February 2008; Revised 10 May 2008; Accepted 22 July 2008

Recommended by Mary Ingram

A further development of the synchronous array method (SAM) as a medium access control scheme for large-scale ad hoc wireless networks is presented. Under SAM, all transmissions of data packets between adjacent nodes are synchronized on a frame-byframe basis, and the spacing between concurrent cochannel transmissions of data packets is properly controlled. An opportunistic SAM (O-SAM) is presented which allows concurrent cochannel transmissions to be locally adaptive to channel gain variations. A distributed SAM (D-SAM) is discussed that schedules all concurrent cochannel transmissions in a distributed fashion. For networks of low mobility, the control overhead required by SAM can be made much smaller than the payload. By analysis and simulation, the intranetwork throughput of O-SAM and D-SAM is evaluated. The effects of traffic load and multiple antennas on the intranetwork throughput are studied. The throughput of ALOHA is also analyzed and compared with that of O-SAM and D-SAM. By a distance-weighted throughput, a comparison of long distance transmission versus short distance transmission is also presented. The study of D-SAM reveals an important insight into the MSH-DSCH protocol adopted in IEEE 802.16 standards.

Copyright () 2009 Yuan Yu et al. This is an open access article distributed under the Creative Commons Attribution License, which permits unrestricted use, distribution, and reproduction in any medium, provided the original work is properly cited.

\section{Introduction}

We consider large-scale ad hoc wireless networks of low mobility within a time interval. Depending on applications, this time interval can be on a time scale of minutes, hours, days, or even longer. Such networks include many types of rapidly deployable wireless networks. There are two types of traffic in ad hoc networks. One is internetwork traffic where traffic flows through one or more gateways (also known as access points) to or from a backbone network. The other is intranetwork traffic where traffic stays within the ad hoc network. For internetwork traffic, the aggregated network throughput is obviously upper bounded by the capacity of the gateways. By either throughput or capacity, we mean network spectral efficiency in terms of bits $/ \mathrm{s} / \mathrm{Hz}$ (bits per second per Hertz). More specifically, we will use bits-hop/s/Hz/node and bits-meter/s/Hz/node as the fundamental intranetwork throughput measure $[1,2]$, which will be explained later. The internetwork traffic will not be considered in this paper.

For intranetwork traffic, the achievable network throughput has been a topic of research by information theorists for many years. A well-known result on this subject is the scaling law shown in [1]. This is also a subject reviewed in [2]. It is arguable that if a network is large in terms of the number of nodes relative to the logarithm of the available transmission power from each node, the network throughput in bits-hop/s/Hz/node (i.e., bits-hop per second per Hertz per node) is upper bounded [2]. Here, bits-hop means the (averaged) number of bits transported from one node to any of its adjacent nodes, and "per node" means "per every source node." This measure of throughput is also a per-link network throughput. If the network node density is denoted by $\rho$, then the distance per hop is in the order of $1 / \rho$ for $1 \mathrm{D}$ network, $1 / \rho^{1 / 2}$ for $2 \mathrm{D}$ network, and $1 / \rho^{1 / 3}$ for $3 \mathrm{D}$ network. If we denote the upper bound of the per-link network throughput by $c$, then the distanceweighted network throughput in bits-meter/s/Hz/node is upper bounded by $c / \rho$ for $1 \mathrm{D}$ network, $c / \rho^{1 / 2}$ for $2 \mathrm{D}$ network, and $c / \rho^{1 / 3}$ for 3D network. Here, bits meter means the number of bits transported over one meter distance. In this paper, we will only consider $2 \mathrm{D}$ networks. The above expression $c / \rho^{1 / 2}$ for $2 \mathrm{D}$ network is equivalent to the capacity scaling law shown in [1] for a $2 \mathrm{D}$ network of arbitrary 


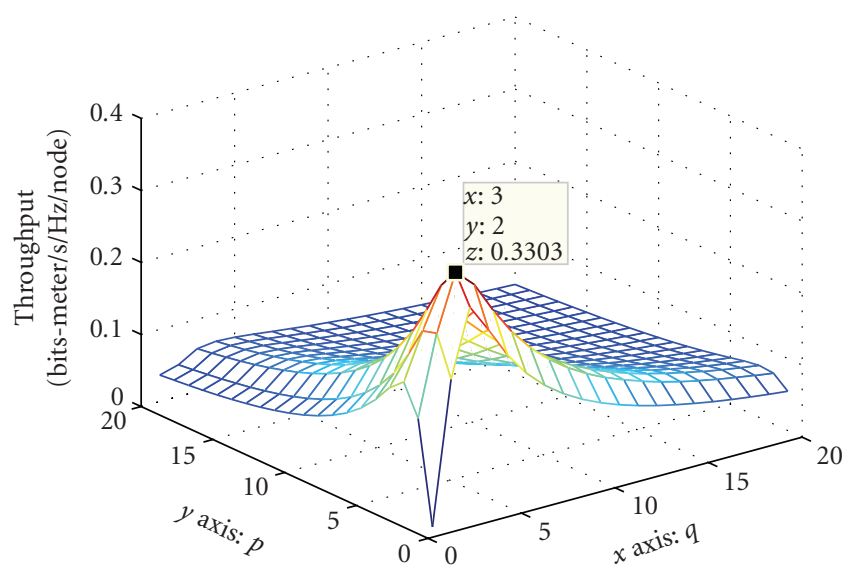

Figure 1: The throughput in bits-meter/s/Hz/node of a large network of 245 nodes on square grid versus $p$ and $q$ in the SAM protocol [2]. The node density is one. The SNR at each receiver is $40 \mathrm{~dB}$. The channel coefficients are constant.

topology where total $n$ nodes are inside a unit-area disk and hence $\rho=n$. It is further shown in [1] that if the network topology is random, then the averaged network throughput has an extra penalty factor in the form of $1 / \sqrt{\log n}$. Since [1], there have been new findings on the capacity scaling laws of large-scale ad hoc networks in various alternative settings [3-9]. It should be noted that although representing a theoretical challenge to the above-stated scaling law, a result shown in [10] requires extremely-large-scale virtual multiple-input-multiple-output (MIMO) channels and is highly infeasible according to our analysis.

The capacity scaling laws as discussed above only reveal the effect of the network size. The exact throughput of a large network depends on a wide range of factors. Among them, medium access control (MAC) is critically important. Most of the existing MAC schemes for ad hoc networks are variations of the two basic forms: ALOHA [11] and CSMA (carrier sense multiple access). With CSMA, a node can transmit a packet only when there is no other concurrent cochannel transmission within a large radius. The per-link throughput of CSMA diminishes to zero as quickly as the inverse of the number of nodes within the carrier sensing radius. It is useful to note however that CSMA is adopted in IEEE 802.11 standards [12] for small single-hop networks. With ALOHA, each node initiates a packet transmission randomly. This packet can be received successfully if the intended receiver is ready and the interference is not too high. Because concurrent cochannel transmissions are allowed by ALOHA, the per link throughput of ALOHA does not reduce to zero as the node density increases. In other words, with ALOHA, the capacity scaling law $c / \rho^{1 / 2}$ in bits meter/s/Hz/node holds for networks of regular topologies. A throughput analysis of ALOHA for large network is available in [13]. The throughput shown in [13] was not maximized over the target SINR $\xi$. As shown in [14], $\xi$ affects the network throughput significantly and can be optimized in practice. In this paper, we distinguish between signal- to-interference-and-noise ratio (SINR) and signal-to-noise ratio (SNR).

For many potential applications, ad hoc networks have low mobility during operations, which allows cooperations that are not exploited by ALOHA. In [2], the synchronous array method (SAM) was proposed. The essence of SAM is to partition all links in the network into multiple interleaved subsets of links where each subset of links with desired spacing between them corresponds to a set of concurrent cochannel transmissions. As an example, Figure 1 illustrates the impact of the spacing between concurrent cochannel transmissions on the network throughput. For this figure, all nodes are on the square grid. For square topology, the spacing or sparseness is measured by $p$ and $q$ which are the vertical and horizontal spacing units between concurrent cochannel transmitters [2]. Also for this figure, the target SINR $\xi$ (i.e., the required SINR value for a packet to be received successfully) is optimized for each pair of $p$ and $q$, the channels are nonfading (complex Gaussian fading channels will be considered in the sequel of this paper), and single omnidirectional antenna is used on each node. We see that the impact of the sparseness is significant. For regular topologies such as square, triangle, and hexagon, the sparseness can also be measured by the ratio $f_{s}$ of the total number of nodes in the network over the number of nodes that are receiving (or transmitting) in each timefrequency slot. In Figure $1, p=2$ and $q=3$ are optimal. The corresponding $f_{s}$ is six. Depending on network topology, antenna properties, and channel fading characteristics, the optimal value of $f_{s}$ varies. But for regular topologies and omnidirectional antennas, the optimal value of $f_{s}$ has been found mostly in the range of four, five, and six [14]. If CSMA is applied, the sparseness of concurrent cochannel transmissions would be very large and the network throughput would be far below the peak value shown in Figure 1. The idea of using concurrent cochannel transmissions to improve the network efficiency is gaining more attention [15].

The analysis in [14] shows that the throughput of SAM is significantly (about two times) higher than that of ALOHA. In [16], an opportunistic SAM (O-SAM) was proposed that allows concurrent cochannel transmissions to be locally adaptive to channel gain variations. This idea is similar to one used in a channel-state-dependent ALOHA [17] for a single-hop network. But the context for O-SAM is a multihop network rather than a single-hop network. Since the strongest channel gain within each local area is exploited each time, the throughput of O-SAM is much improved. The effect of using multiple antennas is also considered in [18]. However, all of the existing throughput analyses of ALOHA, SAM, and O-SAM are under a full loading condition where each node always has a packet waiting to be transmitted at any time.

In this paper, we will present several new contributions. The first is a comparison of ALOHA and O-SAM under a more general loading condition. This condition is modeled as the probability $\zeta$ that each node has a packet for transmission at any given time. We will reveal that the $(\xi$ optimized) throughput of ALOHA is lower than that of OSAM unless $\zeta$ is small (e.g., less than 10\%). The second is a 


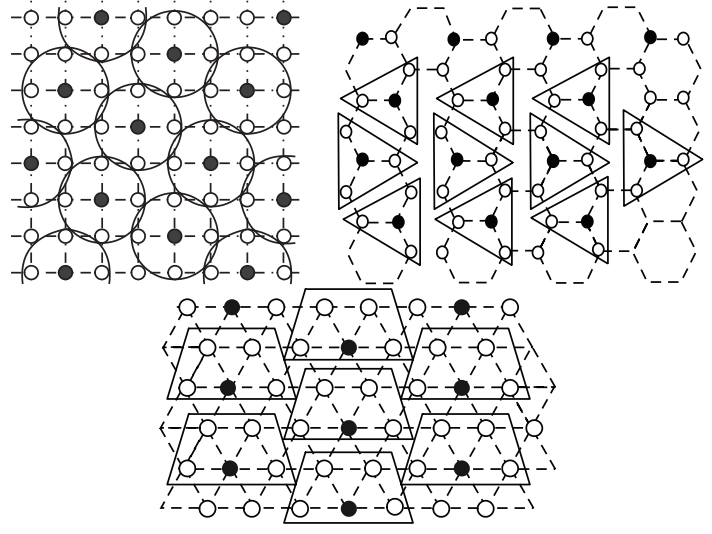

FIGURE 2: Optimal subnet partitions of large networks on regular topologies for O-SAM: the upper left is square, the upper right is hexagon, and the lower is triangle. The sparseness factor $f_{s}$ is five for the square and triangle topologies and four for the hexagonal topology. The black nodes are concurrent cochannel receivers. One of the blank nodes in each subnet can be a transmitter in that subnet.

comparison of longer-distance transmission versus shortestdistance transmission in terms of the distance-weighted throughput in bits-meter/s/Hz/node, which shows that the former is worse than the latter unless $\zeta$ is very small (e.g., less than 1\%). The third is an analysis of O-SAM for the case of multiple antennas on each node, which is an extension of that in $[16,18]$. The forth is an introduction and evaluation of a distributed SAM (D-SAM) which allows all concurrent cochannel transmissions to be scheduled in a distributed and dynamic way. The essence of D-SAM is similar to that of MSH-DSCH in IEEE 802.16 standards [19]. However, there has been no prior study of the fundamental throughput of MSH-DSCH in large networks. The understanding of DSAM for large networks can serve this purpose. The study shown in [20] focuses on the dynamic of control packet exchanges, which as explained later does not reveal the fundamental throughput of a network of low mobility. By simulation, we will show the effect of a cooperative radius $\mathcal{R}$ on the throughput of D-SAM. Within the radius $\mathcal{R}$ centered at a receiver, only the desired transmitter is allowed to transmit a data packet. It is important to note that the cooperative radius $\mathcal{R}$ is smaller than an eavesdropping radius $\mathcal{R}_{e}$. The latter defines the maximum distance between any two nodes which can eavesdrop each other. Furthermore, a carrier sensing radius $\mathcal{R}_{c}$ would be much larger than the eavesdropping radius $\mathcal{R}_{e}$. This study interestingly supports that the two-hop rule adopted in MSH-DSCH (i.e., all interfering transmitters to a receiver are kept two hops away from the receiver) is a good choice for regular or nearregular topologies under a full load condition. This study also provides a corresponding guidance for choosing a proper packet spectral efficiency, which is not available in IEEE 802.16 .

The principle of D-SAM differs from that of a distributed and cooperative link scheduling (DCLS) algorithm shown in [21]. The former is based on the distance information of neighboring nodes. But the latter is based on calibration of actual SINR for each link. For environment where distance does not well reflect signal attenuation, DCLS could be a better alternative. A detailed comparison between D-SAM and DCLS is not yet available.

Whenever feasible, analysis is given. Otherwise, only simulation is provided. We will measure network throughput by bits-meter/s/Hz/node. All numerical examples to be shown are useful fundamental benchmarks for large networks.

The reminder of this paper is organized as follows. In Section 2, we extend O-SAM presented in [18] by taking into account the loading probability $\zeta$. In Section 3, we analyze the network throughput of O-SAM, where the single-inputsingle-output (SISO), single-input-multiple-output (SIMO), and multiple-input-multiple-output (MIMO) channels are all considered. In Section 4, we present D-SAM in detail. In Section 5, we revisit the slotted ALOHA with consideration of the loading probability $\zeta$. In Section 6, we evaluate and compare the network throughput of ALOHA, O-SAM, and D-SAM.

\section{Opportunistic SAM}

2.1. Subnet Partitions. As mentioned before, the essence of SAM proposed in [2] is to partition all links in the network into multiple interleaved subsets of links where each subset of links with desired spacing between them corresponds to a set of concurrent cochannel transmissions. An equivalent description of SAM is that in any given time-frequency slot, the entire network is partitioned into contiguous subnets and each subnet consists of a receiving node, a transmitting node and possibly several idle nodes. In different time-frequency slots, the corresponding partitions of subnets are relatively shifted from each other.

Figure 2 illustrates the partitions of subsets for square, triangle and hexagonal topologies. For opportunistic SAM (O-SAM), each receiving node is chosen to be at the center of each subnet, and the transmitting node in each subnet is opportunistically selected from other nodes in the subnet. This is different from SAM in [2] which will also be referred to as centralized SAM (C-SAM) where both receiving and transmitting nodes in each subnet are predetermined.

For the O-SAM protocol shown next and the Gaussian fading channels, the subnet partitions shown in Figure 2 have been found to be optimal among other possible partitions. It is useful to note that except for the hexagonal topology, the subnet partitions shown in this figure are not exactly the same as the optimal ones for C-SAM as shown in [14]. But the fact that the optimal subnet partition for the hexagonal topology is the same for both C-SAM and O-SAM makes the hexagonal topology more interesting. This is because the throughput gain by O-SAM via opportunistic selection of transmitters is no longer compromised by altering the subnet partition from the optimal one determined by C-SAM. This advantage will be illustrated numerically later.

2.2. The Protocol. The O-SAM protocol is described next. Without loss of generality, we can focus on a single 
time-frequency frame. For a large network, almost all subnets can be treated like a subnet in the center of the network. We will refer to such a subnet as subnet 0 and any other subnet as subnet $j$ with $j=1,2, \ldots, S$. We let $I_{0}$ denote the set of the indices of all potential transmitting nodes that have packets to transmit to the receiver in subnet 0 . We let $n_{0}$ be the total number of nodes, other than the receiver, in subnet 0 . Since $\zeta$ is the probability that a node has a packet to transmit to another node, the probability that $I_{0}$ contains $k$ nodes is $\zeta^{k}(1-\zeta)^{n_{0}-k}$. Note that the set $I_{0}$ is a random set in each time-frequency frame.

2.2.1. SISO Channels. If the channel between every two nodes is modeled as SISO channel, the channel coefficient from the $i$ th node in $I_{0}$ to its receiver is denoted by $h_{0,0}(i)$. The corresponding channel gain is $v_{0,0}(i)=\left|h_{0,0}(i)\right|^{2}$. The index of the node with the strongest gain in subnet 0 is denoted by $i_{0, \max }=\arg \max _{i \in I_{0}} v_{0,0}(i)$. The index of the node selected for transmission in subnet 0 is

$$
k_{0}= \begin{cases}i_{0, \max } & \text { if } v_{0,0}\left(i_{0, \max }\right) \geq \theta, \\ \{\varnothing\} & \text { otherwise. }\end{cases}
$$

Here, no node is selected for transmission in subnet 0 if the gain of the node with the strongest gain in the subnet is less than a prespecified threshold $\theta$. The reason behind the use of $\theta$ is that if the strongest gain in a subnet is too small, abandoning packet transmission in this subnet causes little loss of information in this subnet and at the same time reduces interference to other subnets. A significant impact of $\theta$ on the network throughput under a full load condition was illustrated in [16].

The O-SAM protocol (1) requires each subnet to know the channel gains of all potential transmitting nodes in the subnet. This requires channel estimation and associated exchanges of control packets. This task is feasible if the channel coherence time is relatively long. In fact, for networks of low mobility, the channel coherence time can be very large (e.g., many milliseconds). In this case, only a small fraction (e.g., a few micro seconds) of the channel coherence time needs to be spent for channel estimation. Clearly, the more coordinated is the channel estimation in all subnets, the less time is needed. We will not further address the implementation issues of channel estimation for O-SAM.

On the other hand, if the channel gains do not change over time, there is no opportunity to be exploited by O-SAM and the protocol (1) is not meaningful. But random changes in channel gains can be induced artificially if they are not present naturally. To induce random channel gains, one can use multiple transmit antennas on each node and choose a transmit beam vector for each node randomly from frame to frame. This technique also applies to the SIMO and MIMO cases discussed below. The key is to compress the dimension of the channel responses randomly at the transmitter side.

2.2.2. SIMO Channels. If each transmitting node uses one antenna and each receiving node uses multiple antennas, we have a SIMO channel between each transmitter and its receiver. In this case, we define the O-SAM protocol as (1) except that we use $v_{0,0}(i)=\left\|\mathbf{h}_{0,0}(i)\right\|^{2}$ where $\mathbf{h}_{0,0}(i)$ is the channel response vector between the $i$ th node in $I_{0}$ and its receiver.

We will skip the MISO case since it is similar to the SIMO case.

2.2.3. MIMO Channels. If each node has multiple transmit antennas and multiple receive antennas, we have a MIMO channel between each transmitter and its receiver. In this case, we define the O-SAM protocol as (1) except that $v_{0,0}(i)=\lambda_{\max }\left(\mathbf{H}_{0,0}(i) \mathbf{H}_{0,0}^{H}(i)\right)$ where $\lambda_{\max }$ denotes the largest eigenvalue and $\mathbf{H}_{0,0}(i)$ is the channel response matrix between the $i$ th node in $I_{0}$ and its receiver. The use of $\lambda_{\max }$ implies that the principal stream of each MIMO channel is used but all other streams are ignored. Because of the interference between concurrent cochannel transmissions, the inclusion of the nonprincipal streams of each MIMO channel into the O-SAM protocol would make the throughput analysis intractable to us at this stage. For this reason, we only consider the principal stream of each MIMO channel.

\section{Throughput Analysis of Opportunistic SAM}

For throughput analysis, we assume that all elements in channel coefficients, channel response vectors, and channel response matrices are independent and identically distributed (i.i.d.) complex Gaussian random variables. This implies in particular that the channel coefficient between any receive antenna and any transmit antenna is independent of all other channel coefficients.

3.1. SISO Channels. For SISO channels, the signal $y_{0}$ received by the receiving node in subnet 0 can be written as

$$
y_{0}=h_{0,0} x_{0}+\sum_{j>0} h_{0, j} x_{j}+w_{0},
$$

where $x_{j}$ is the transmitted signal from the transmitter in subnet $j, h_{0, j}$ is the channel coefficient between the transmitter in subnet $j$ and the receiver in subnet 0 , and $w_{0}$ is white Gaussian noise with zero mean and variance $\sigma^{2}$. We assume that $h_{0, j}$ is complex Gaussian random variable (from frame to frame) with zero mean and variance $E\left|h_{0, j}\right|^{2}=d_{0, j}^{-\alpha}$. Here, $\alpha$ is the path loss exponent and $d_{0, j}$ is the distance between the transmitter and the receiver. For convenience of analysis, we assume that all nodes transmit with the same power $P$, that is, $E\left|x_{j}\right|^{2}=P$. Hence, the instantaneous SINR at the receiver in subnet 0 is

$$
\operatorname{SINR}=\frac{v_{0,0} P}{\sum_{j>0} v_{0, j} P+\sigma^{2}},
$$

where $v_{0,0}=\left|h_{0,0}\right|^{2}$ and $v_{0, j}=\left|h_{0, j}\right|^{2}$. We assume that the instantaneous SINR at each receiver is not known to the desired transmitter, which is due to random transmissions from other subnets. We also assume that for a large network, almost all the subnets are statistically equivalent 
to each other. Then, the network throughput in bitsmeter/s/Hz/node can be expressed as

$$
C_{\mathrm{O}-\mathrm{SAM}}=\frac{\beta}{L \sqrt{\rho}} R_{\xi} P_{d}
$$

where $R_{\xi}=\log _{2}(1+\xi)$ is the packet spectral efficiency, and $P_{d}=\operatorname{Pr}\{\operatorname{SINR} \geq \xi\}$ is the probability of a successful packet detection. Also, $L$ is the node population in each subnet. As illustrated in Figure 2, $L=5$ for the square and triangle topologies, and $L=4$ for the hexagonal topology. Finally, $\beta$ is a conversion factor from bits/hop/s/Hz/node to bitsmeter/s/Hz/node. As shown in [14], we have $\beta=0.785$ for square topology, $\beta=0.689$ for hexagonal topology, and $\beta=$ 0.975 for triangle topology. Strictly speaking, the expression (4) is the network throughput for the interior region of the network. For large networks, (4) is a tight lower bound on the network throughput for the entire network.

From the O-SAM protocol, it is clear that the instantaneous SINR in each time-frequency frame is a random variable that depends on $\theta$ and $\zeta$, and hence the network throughput is effected by $\xi, \theta$, and $\zeta$.

In order to evaluate the network throughput (4), we need a more explicit form of $P_{d}$, which is derived next:

$$
\begin{aligned}
P_{d} & =\operatorname{Pr}\left\{\operatorname{SINR} \geq \xi, v_{0,0} \geq \theta\right\} \\
& =\operatorname{Pr}\left\{v_{0,0} \geq \xi\left(\frac{\sigma^{2}}{P}+\sum_{j>0} v_{0, j}\right), v_{0,0} \geq \theta\right\} \\
& =\operatorname{Pr}\left\{\sum_{j>0} v_{0, j} \leq \frac{v_{0,0}}{\xi}-\frac{\sigma^{2}}{P}, v_{0,0} \geq \theta\right\} \\
& =\int_{\max \left(\xi \sigma^{2} / P, \theta\right)}^{\infty}\left(\int_{0}^{y / \xi-\sigma^{2} / P} f_{v_{I}}(x) d x\right) f_{v_{0,0}}(y) d y,
\end{aligned}
$$

where $f_{v_{0,0}}(y)$ is the probability density function (pdf) of $v_{0,0}$, and $f_{v_{I}}(x)$ is the pdf of $v_{I}=\sum_{j>0} v_{0, j}$. Note that the condition $v_{0,0} \geq \theta$ in the above expression is important. The impact of $\theta$ on the network throughput is significant and illustrated in [16] (under the full load condition). In this paper, we will not further illustrate the effect of $\theta$. Unless mentioned otherwise, $\theta$ is optimally chosen to maximize the network throughput.

In order to evaluate $P_{d}$ shown in (5), we need to obtain the expressions of the two pdf functions $f_{v_{0,0}}(y)$ and $f_{v_{I}}(x)$. We start with $f_{v_{0,0}}(y)$. Since $\left|h_{0,0}(m)\right|^{2}$ is exponentially distributed with the mean $D_{0,0}(m)=d_{0,0}^{-\alpha}(m)$, where $d_{0,0}(m)$ is the distance between the transmitter and receiver in subnet 0 and $\alpha$ is the path loss exponent, it follows that

$$
\begin{aligned}
\operatorname{Pr}\left\{v_{0,0} \leq y\right\} & =\prod_{m \in I_{0}} \operatorname{Pr}\left\{v_{0,0}(m) \leq y\right\} \\
& =\prod_{m \in I_{0}}\left(1-\exp \left\{\frac{-y}{D_{0,0}(m)}\right\}\right) U(y),
\end{aligned}
$$

where $U(y)$ is the unit step function. The above expression is not ready to use since $I_{0}$ is a random set. Alternatively and equivalently, we can think of a node that has no packet to transmit as if it is a node that has zero channel gain with respect to the receiver. Following this thinking, we can write

$$
\begin{aligned}
\operatorname{Pr}\left\{v_{0,0} \leq y\right\} & =\prod_{m=1}^{n_{0}}\left\{\left(1-\exp \left\{\frac{-y}{D_{0,0}(m)}\right\}\right) \zeta+(1-\zeta)\right\} U(y) \\
& =\prod_{m=1}^{n_{0}}\left(1-\zeta \exp \left\{\frac{-y}{D_{0,0}(m)}\right\}\right) U(y),
\end{aligned}
$$

where $n_{0}$ is the number of potential transmitters in subnet 0 . The pdf $f_{v_{0,0}}(y)$ follows readily from the derivative of $\operatorname{Pr}\left\{v_{0,0} \leq y\right\}$ shown in (7), that is,

$$
\begin{aligned}
f_{v_{0,0}}(y)=\sum_{k=1}^{n_{0}} & \left(\delta(y)+\zeta \frac{1}{D_{0,0}(k)} \exp \left\{\frac{-y}{D_{0,0}(k)}\right\}\right) \\
& \times \prod_{m=1, m \neq k}^{n_{0}}\left(1-\zeta \exp \left\{\frac{-y}{D_{0,0}(m)}\right\}\right) U(y),
\end{aligned}
$$

where $\delta(y)$ is the Dirac's delta function.

To derive the pdf $f_{v_{I}}(x)$ where $v_{I}=\sum_{j>0} v_{0, j}$, we start with the following:

$$
\begin{aligned}
\operatorname{Pr}\left\{v_{0, j} \leq x\right\} & =\left\{\overline{P_{j}}+\sum_{l=1}^{n_{j}} P_{j, l} \operatorname{Pr}\left\{\left|h_{0, j}(l)\right|^{2} \leq x\right\}\right\} U(x) \\
& =\left\{\overline{P_{j}}+\sum_{l=1}^{n_{j}} P_{j, l}\left(1-\exp \left\{\frac{-x}{D_{0, j}(l)}\right\}\right)\right\} U(x),
\end{aligned}
$$

where $\overline{P_{j}}$ is the probability that there is no transmission in subnet $j$, and $P_{j, l}$ is the probability that the $l$ th node in subnet $j$ transmits. We have used $n_{j}$ to denote the number of potential transmitters in subnet $j$. In (9), we also used the property that $\left|h_{0, j}(l)\right|^{2}$ is exponentially distributed with the mean $D_{0, j}(l)=d_{0, j}^{-\alpha}(l)$, where $d_{0, j}(l)$ is the distance between the $l$ th transmitter in subnet $j$ and the receiver in subnet 0 . It follows that

$$
\begin{aligned}
\overline{P_{j}} & =1-\sum_{l} P_{j, l} \\
P_{j, l} & =\zeta \cdot \operatorname{Pr}\left\{v_{j, j}(l) \geq \theta, \max _{k \neq l, k \in I_{j}} v_{j, j}(k) \leq v_{j, j}(l)\right\} \\
& =\zeta \int_{\theta}^{\infty} \frac{1}{D_{j, j}(l)} e^{-x / D_{j, j}(l)} \prod_{k \neq l, k \in\left\{1,2, \ldots, n_{j}\right\}}\left(1-\zeta e^{-x / D_{j, j}(k)}\right) d x,
\end{aligned}
$$

where we have used the technique used for (7). Then, the pdf $f_{v_{0, j}}(x)$ follows readily from the derivative of $(9)$, which is a superimposed-exponential, that is,

$$
f_{v_{0, j}}(x)=\overline{P_{j}} \delta(x)+\sum_{l=1}^{n_{j}} P_{j, l} \frac{1}{D_{0, j}(l)} e^{-x / D_{0, j}(l)} U(x) .
$$

Since $v_{I}$ is the sum of the independent random variables $v_{0, j}$ for all $j>0$, the pdf of $f_{v_{I}}(x)$ is the convolution of $f_{v_{0, j}}(x)$ 
for all $j>0$. Assume that $f_{v_{I}}(x)$ is negligible for $x \geq T$. We can write the Fourier series expansion of $f_{v_{I}}(x)$ as follows:

$$
f_{v_{I}}(x)=\sum_{k=-K}^{K} g_{k} \exp \left(v \frac{2 \pi k}{T} x\right)
$$

where $v=\sqrt{-1}$ and

$$
\begin{aligned}
g_{k} & =\frac{1}{T} \int_{0}^{T} f_{v_{I}}(t) \exp \left(-v \frac{2 \pi k}{T} t\right) d t \\
& =\frac{1}{T} \prod_{j>0}\left(\overline{P_{j}}+\sum_{l=1}^{n_{j}} \frac{P_{j}^{l}}{1+v(2 \pi k / T) D_{0, j}(l)}\right) .
\end{aligned}
$$

We will assume that $g_{k}$ is negligible for $k>K$.

With $f_{v_{0,0}}(x)$ and $f_{v_{I}}(x)$ as shown above, $P_{d}$ in (5) can be readily computed.

3.2. SIMO Channels. For SIMO channels where there are $n_{r}$ receiving antennas at each node, the signal received by the receiver in subnet 0 has the following expression:

$$
\mathbf{y}_{0}=\mathbf{h}_{0,0} x_{0}+\sum_{j>0} \mathbf{h}_{0, j} x_{j}+\mathbf{w}_{0}
$$

Here, $x_{i}$ denotes the signal transmitted from subnet $i$. $\mathbf{h}_{i, j} \in \mathbb{C}^{n_{r} \times 1}$ is the channel coefficient vector between the transmitter in subnet $j$ and the receiver in subnet $i$. The entries in $\mathbf{h}_{i, j}$ are assumed to be independent and identically distributed complex Gaussian random variables with zero mean and variance $d_{i, j}^{-\alpha}\left(k_{j}\right)$ where $k_{j}$ is given by (15) and $d_{i, j}\left(k_{j}\right)$ is the distance between the transmitter in subnet $j$ and the receiver in subnet $i, \alpha$ is the path loss exponent. $\mathbf{w}_{i}$ is the complex noise vector at the receiver in subnet $i$, and assumed to have zero mean and the covariance matrix $E\left\{\mathbf{w}_{i}^{H} \mathbf{w}_{i}\right\}=\sigma^{2} \mathbf{I}_{n_{r}}$ where $\mathbf{I}_{n_{r}}$ denotes the $n_{r} \times n_{r}$ identity matrix. We also assume that all the nodes in the network transmit with the same power $P$, that is, $E\left\{x_{i}^{H} x_{i}\right\}=P$.

It is important to note that based on the O-SAM protocol, $\mathbf{h}_{i, j}=\mathbf{h}_{i, j}\left(k_{j}\right)$ where

$$
k_{j}=\arg \left(\max _{k \in I_{j},\left\|\mathbf{h}_{j, j}(k)\right\|^{2} \geq \theta}\left\|\mathbf{h}_{j, j}(k)\right\|^{2}\right) .
$$

Also recall that $\mathbf{h}_{i, j}(k)$ is the channel response vector from the $k$ th potential transmitter in subnet $j$ to the receiver in subnet $i$.

A sufficient statistics of $\mathbf{y}_{0}$ is given by $r_{0}=\mathbf{h}_{0,0}^{H} \mathbf{y}_{0}$. The SINR in $r_{0}$ is

$$
\begin{aligned}
\operatorname{SINR} & =\frac{\mathbf{h}_{0,0}^{H} \mathbf{h}_{0,0}}{\sum_{j>0}\left(\left|\mathbf{h}_{0,0}^{H} \mathbf{h}_{0, j}\right|^{2} /\left\|\mathbf{h}_{0,0}\right\|^{2}\right)+\sigma^{2} / P} \\
& =\frac{v_{0,0}}{\sum_{j>0} v_{0, j}+\sigma^{2} / P},
\end{aligned}
$$

where $v_{0,0}=\mathbf{h}_{0,0}^{H} \mathbf{h}_{0,0}$ and $v_{0, j}=\left|\mathbf{h}_{0,0}^{H} \mathbf{h}_{0, j}\right|^{2} /\left\|\mathbf{h}_{0,0}\right\|^{2}$.

Given any $\mathbf{h}_{0,0}, h_{j}(l) \doteq \mathbf{h}_{0,0}^{H} \mathbf{h}_{0, j}(l) /\left\|\mathbf{h}_{0,0}\right\|$ is a linear combination of the elements of $\mathbf{h}_{0, j}(l)$ which are i.i.d. complex Gaussian random variable, and hence $h_{j}(l)$ is a complex Gaussian variable. Each element of $\mathbf{h}_{0, j}(l)$ has zero mean and the variance $D_{0, j}(l)=d_{0, j}^{\alpha}(l)$ where $d_{0, j}(l)$ is the distance between the $l$ th node in subnet $j$ and the receiver in subnet 0 . Furthermore, one can verify as in [22] that $h_{j}(l)$ has zero mean and the variance $D_{0, j}(l)$. It follows that $v_{0, j}(l)=\left|h_{j}(l)\right|^{2}$ for $j>0$ is independent of $\mathbf{h}_{0,0}$ and is exponentially distributed with mean $D_{0, j}(l)$, that is,

$$
f_{v_{0, j}(l)}(y)=\frac{1}{D_{0, j}(l)} \exp \left(\frac{-y}{D_{0, j}(l)}\right) U(y), \quad j>0 .
$$

Since $v_{0, j}=v_{0, j}\left(k_{j}\right)$ with $k_{j}$ given by $(15), v_{0, j}$ for $j>0$ is also independent of $\mathbf{h}_{0,0}$.

Therefore, with the above description of $v_{0,0}$ and $v_{0, j}$, the throughput expression (4) and the probability-of-detection expression (5) are also valid for the case of SIMO channels except that the expressions of the pdf $f_{v_{0,0}}(y)$ of $v_{0,0}$ and the pdf $f_{v_{I}}(x)$ of $v_{I}=\sum_{j>0} v_{0, j}$ need to be revised as follows.

To find $f_{v_{0,0}}(y)$, we first write

$$
\operatorname{Pr}\left\{v_{0,0} \leq y\right\}=\prod_{m=1}^{n_{0}}\left\{\zeta \operatorname{Pr}\left(\left\|\mathbf{h}_{0,0}(m)\right\|^{2} \leq y\right)+(1-\zeta)\right\} U(y)
$$

It is known that $\left\|\mathbf{h}_{0, j}(l)\right\|^{2}$ is Chi-square or gamma distributed with $2 n_{r}$ degrees, that is,

$$
f_{\left\|\mathbf{h}_{0, j}(l)\right\|^{2}}(x)=\frac{x^{n_{r}-1}}{\left(n_{r}-1\right) ! D_{0, j}^{n_{r}}(l)} e^{-x / D_{0, j}(l)} U(x) .
$$

Therefore,

$$
\begin{aligned}
\operatorname{Pr}\left\{v_{0,0} \leq y\right\}= & \prod_{m=1}^{n_{0}}\left\{\zeta \int_{0}^{y} \frac{x^{n_{r}-1}}{\left(n_{r}-1\right) ! D_{0,0}^{n_{r}}(m)} e^{-x / D_{0,0}(m)} d x\right. \\
& +(1-\zeta)\} U(y) \\
= & \prod_{m=1}^{n_{0}}\left\{\zeta\left(1-e^{-y / D_{0,0}(m)} \sum_{k=0}^{n_{r}-1} \frac{y^{k}}{D_{0,0}^{k}(m) k !}\right)\right. \\
& \quad+1-\zeta\} U(y) \\
= & \prod_{m=1}^{n_{0}}\left\{1-\zeta e^{-y / D_{0,0}(m)} g\left(\frac{y}{D_{0,0}(m)}\right)\right\} U(y),
\end{aligned}
$$

where $g(y)=\sum_{k=0}^{n_{r}-1}\left(y^{k} / k !\right)$. The pdf $f_{v_{0,0}}(y)$ is simply given by the derivative of $(20)$. If all potential transmitters in each subnet have the same distance to the receiver in the same 
subnet, that is, $D_{j, j}(m)=D$ for all $j$ and all $m$, the pdf $f_{v_{0,0}}(y)$ can be shown to be

$$
\begin{aligned}
f_{v_{0,0}}(y)= & \frac{\partial}{\partial y} \operatorname{Pr}\left\{v_{0,0} \leq y\right\} \\
= & \sum_{\mu=1}^{n_{0}}\left(\begin{array}{c}
n_{0} \\
\mu
\end{array}\right)(-1)^{\mu+1} \zeta^{\mu} e^{-(\mu / D) y} g^{\mu-1}\left(\frac{y}{D}\right) \\
& \times \frac{\mu y^{n_{r}-1}}{D^{n_{r}}\left(n_{r}-1\right) !} U(y)+(1-\zeta)^{n_{0}} \delta(y) \\
= & n_{0}\left[1-\zeta e^{-y / D} g\left(\frac{y}{D}\right)\right]^{n_{0}-1} \frac{\zeta e^{-y / D} y^{n_{r}-1}}{D^{n_{r}}\left(n_{r}-1\right) !} U(y) \\
& +(1-\zeta)^{n_{0}} \delta(y) .
\end{aligned}
$$

We now derive the pdf $f_{v_{I}}(x)$ of $v_{I}=\sum_{j>0} v_{0, j}$ where $v_{0, j}=\left|\mathbf{h}_{0,0}^{H} \mathbf{h}_{0, j}\right|^{2} /\left\|\mathbf{h}_{0,0}\right\|^{2}$. Since the pdf of $v_{0, j}(l)$ is the same as that for the SISO channels, all expressions for the pdf $f_{v_{I}}(x)$ are the same as for the SISO case except that $P_{j, l}$ needs to be revised as follows:

$$
\begin{aligned}
P_{j, l}=\zeta \cdot \operatorname{Pr}\left\{\left\|\mathbf{h}_{j, j}(l)\right\|^{2} \geq \max _{k \neq l}\left\|\mathbf{h}_{j, j}(k)\right\|^{2},\left\|\mathbf{h}_{j, j}(l)\right\|^{2} \geq \theta\right\} \\
=\zeta \int_{\theta}^{\infty} \frac{x^{n_{r}-1}}{\left(n_{r}-1\right) ! D_{j, j}^{n_{r}}(l)} e^{-x / D_{j, j}(l)} \\
\quad \times \prod_{k \neq l}\left(1-\zeta e^{-x / D_{j, j}(k)} \sum_{q=0}^{n_{r}-1} \frac{x^{q}}{D_{j, j}(k)^{q} q !}\right) d x,
\end{aligned}
$$

where we have used (19). If $D_{j, j}(l)=D$ for all $j$ and all $l$, then $P_{j, l}$ becomes independent of $l$, and $P_{j, l}$ can be simplified as

$$
P_{j, l}=\zeta \int_{\theta}^{\infty} \frac{x^{n_{r}-1}}{\left(n_{r}-1\right) ! D^{n_{r}}} e^{-x / D}\left(1-\zeta e^{-x / D} \sum_{q=0}^{n_{r}-1} \frac{x^{q}}{D^{q} q !}\right)^{n_{0}-1} d x
$$

3.3. MIMO Channels. For MIMO channels, the received signal model in subnet 0 is given by

$$
\mathbf{y}_{0}=\mathbf{H}_{0,0} \mathbf{x}_{0}+\sum_{i>0} \mathbf{H}_{0, i} \mathbf{x}_{i}+\mathbf{w}_{0}
$$

where $\mathbf{H}_{i, j} \in \mathbb{C}^{n_{r} \times n_{t}}$ is the channel coefficient vector between the transmitter in subnet $j$ and the receiver in subnet $i$. The entries in $\mathbf{H}_{i, j}$ are assumed to be independent and identically distributed complex Gaussian random variables with zero mean and variance $d_{i, j}^{-\alpha}\left(k_{j}\right)$ with $k_{j}$ defined by (25). $\mathbf{x}_{i}$ is the complex vector signal transmitted from subnet $i . \mathbf{w}_{i}$ is the complex noise vector at the receiver in subnet $i$, and assumed to have zero mean and the covariance matrix $E\left\{\mathbf{w}_{i}^{H} \mathbf{w}_{i}\right\}=$ $\sigma^{2} I_{n_{r}}$, where $I_{n_{r}}$ denotes the $n_{r} \times n_{r}$ identity matrix. We also assume that all the nodes in the network transmit with the same power $P$, that is, $\operatorname{tr}\left\{E\left\{\mathbf{x}_{i} \mathbf{x}_{i}^{H}\right\}\right\}=P$. We further assume that $n_{r}=n_{t}$.
Based on the O-SAM protocol, $\mathbf{H}_{i, j}=\mathbf{H}_{i, j}\left(k_{j}\right)$ where

$$
k_{j}=\arg \left(\max _{k \in I_{j}, \lambda_{\max }\left(\mathbf{H}_{i, j}(k)\right) \geq \theta} \lambda_{\max }\left(\mathbf{H}_{i, j}(k)\right)\right) .
$$

Denote the singular value decomposition (SVD) of $\mathbf{H}_{i, i}$ as $\mathbf{H}_{i, i}=\mathbf{U}_{i, i} \Lambda_{i, i}^{1 / 2} \mathbf{V}_{i, i}^{H}$ where $\Lambda_{i, i}$ is a diagonal matrix of nonnegative entries in a descending order. Then, we can transform (24) to the following:

$$
\tilde{\mathbf{y}}_{0}=\Lambda_{0,0}^{1 / 2} \widetilde{\mathbf{x}}_{0}+\sum_{i>0} \tilde{\mathbf{H}}_{0, i} \tilde{\mathbf{x}}_{i}+\tilde{\mathbf{w}}_{0}
$$

where $\tilde{\mathbf{y}}_{0}=\mathbf{U}_{0,0}^{H} \mathbf{y}_{0}, \widetilde{\mathbf{x}}_{i}=\mathbf{V}_{i, i}^{H} \mathbf{x}_{i}, \widetilde{\mathbf{H}}_{0, i}=\mathbf{U}_{0,0}^{H} \mathbf{H}_{0, i} \mathbf{V}_{i, i}$ and $\widetilde{\mathbf{w}}_{0}=$ $\mathbf{U}_{0,0}^{H} \mathbf{w}_{0}$.

Under the O-SAM protocol, we only use the principal stream of each MIMO link. In this case, only the first entry of the vector $\tilde{\mathbf{x}}_{i}$ is nonzero, which is denoted by $x_{i}$. Therefore, a sufficient statistics of the vector $\tilde{\mathbf{y}}_{0}$ is given by its first element, which is denoted by $y_{0}$, and (26) is equivalent to the scalar equation

$$
y_{0}=\lambda_{\max }^{1 / 2} x_{0}+\sum_{j>0} h_{0, j} x_{j}+w_{0}
$$

where $\lambda_{\text {max }}$ is the largest eigenvalue of $\mathbf{H}_{0,0} \mathbf{H}_{0,0}^{H}$, and $h_{0, j}$ is the upper-left entry of $\tilde{\mathbf{H}}_{0, j}$ which is complex Gaussian with zero mean and variance $d_{0, j}^{-\alpha}\left(k_{j}\right)$.

The SINR in $y_{0}$ is given by

$$
\operatorname{SINR}=\frac{v_{0,0}}{\sum_{j>0} v_{0, j}+\sigma^{2} / P},
$$

where $v_{0,0}=\lambda_{\max }$, and $v_{0, j}=\left|h_{0, j}\right|^{2}$ which is exponentially distributed with the mean $D_{0, j}\left(k_{j}\right)=d_{0, j}^{-\alpha}\left(k_{j}\right)$.

Assuming that $d_{0,0}(m)=1$ for all $m=1,2, \ldots, n_{0}$, the cdf (cumulative distribution function) of $\lambda_{\max }$ is known [23] to be

$$
F_{\lambda_{\max }}(x)=\frac{1}{\prod_{j=1}^{n_{r}} \Gamma(j)^{2}} \operatorname{det}\left|\left(\gamma_{i+j-2}\right)\right|,
$$

where $\left(\gamma_{i+j-2}\right)$ is a $n_{r} \times n_{r}$ matrix with element $\gamma_{i+j-2}=$ $\int_{0}^{x} \omega^{i+j-2} \exp (-\omega) d \omega$ and $\Gamma(j)=(j-1)$ !.

The expressions (4) and (5) still hold for the MIMO case except that $f_{v_{I}}(x)$ and $f_{v_{0,0}}(y)$ need to be revised as follows.

We know that

$$
\begin{aligned}
\operatorname{Pr}\left\{v_{0,0} \leq y\right\} & =\prod_{m=1}^{n_{0}}\left\{\zeta F_{\lambda_{\max }}(y)+(1-\zeta)\right\} \\
& =\left\{\zeta F_{\lambda_{\max }}(y)+(1-\zeta)\right\}^{n_{0}} .
\end{aligned}
$$

Then, $f_{v_{0,0}}(y)$ is given by the derivative of (30).

The expressions for $f_{v_{I}}(x)$ are the same as those for the SISO and SIMO cases except that

$$
\begin{aligned}
P_{j, l} & =\frac{1}{n_{j}}\left(1-\overline{P_{j}}\right), \\
\overline{P_{j}} & =\left(1-\zeta+\zeta F_{\lambda_{\max }}(\theta)\right)^{n_{j}} .
\end{aligned}
$$




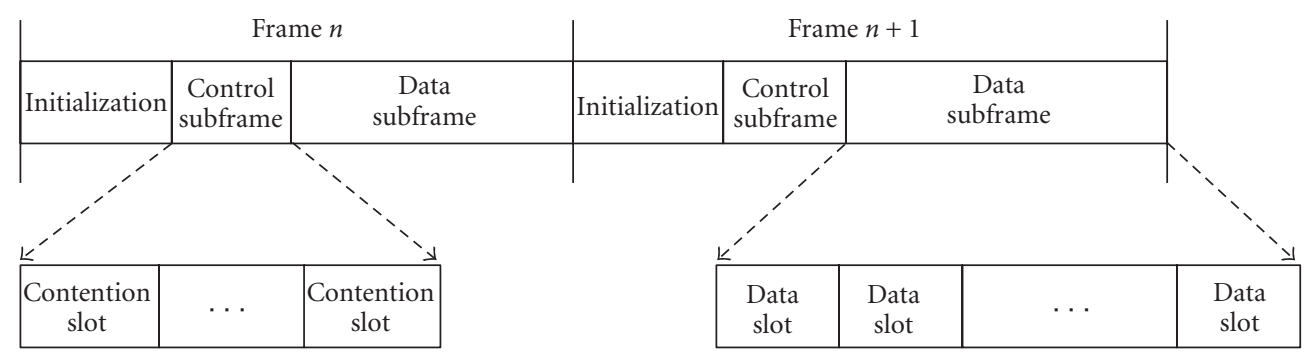

FIgURE 3: Frame structure of the distributed SAM protocol, which resembles that of MSH-DSCH in IEEE 802.16.

\section{Distributed SAM}

The (centralized) SAM shown in [2] and the opportunistic SAM shown in Section 2 all require a subnet partition in a centralized fashion. The dimension of each subnet or the spacing between concurrent cochannel transmissions is critical for network throughput. In this section, we present a distributed SAM (D-SAM) that encapsulates an essence of SAM in that all concurrent cochannel transmissions are properly spaced from each other. But D-SAM forms subnets in each time frame in a distributed and dynamic fashion. DSAM also works with random network topology.

In D-SAM, time is slotted into frames of equal duration as shown in Figure 3. Each frame is further divided into control subframe and data subframe. Assuming that the data subframe is much longer than the control subframe, the network spectral efficiency is dominated by the spectral efficiency in each data subframe. A control subframe is used for each node to compete for data transmission opportunity in a data subframe. Each control subframe consists of a group of $M$ contention slots. For analysis of maximal achievable throughput, we will assume without loss of generality that each data subframe consists of a single data slot.

At the beginning of each frame, D-SAM allows each node to randomly initialize a choice for one of the $M$ contention slots if the node has a packet to transmit to another node. If the node has packets to be transmitted (separately) to multiple neighboring nodes, the node chooses multiple contention slots, one slot for each receiver. During a chosen contention slot, the node contends for the upcoming data subframe by starting a handshaking process with its intended receiving node. The handshaking involves three packets: request-to-sent (RTS), clear-to-sent (CTS), and ACK. If the handshaking is successful, the upcoming data subframe is reserved for data transmission between the transmitter-andreceiver pair. During each contention slot, the handshaking packets are received by neighboring nodes so that these nodes are aware of the reservation status of the upcoming data frame. For each frame and each neighborhood in a predetermined range, the data subframe can only be reserved for one transmitter-and-receiver pair. This means that the first contention slot has the highest priority, the second contention slot has the second priority, and so on. In the next frame, the contention process repeats without memory of the previous contentions, which ensures fairness.
More details of D-SAM are as follows. We assume that each node $k$ maintains a neighborhood list $\mathcal{N}_{k}$ which contains the identifications of all its neighboring nodes inside a cooperative range $\mathcal{R}$. The range $\mathcal{R}$ is an important parameter for the performance of D-SAM. The $i$ th node in $\mathcal{N}_{k}$ is indexed by $\mathcal{N}_{k}(i)$. The neighborhood list at each node can be established at the startup of the network. For networks of low mobility, this startup is feasible. We assume that every node can be set to one of three states for the upcoming data subframe: $T, R$, and $S$. Here, $T$ stands for transmitting, $R$ for receiving, and $S$ for standby. We denote the state of node $k$ as $\S_{k}$ and the state of $\mathcal{N}_{k}(i)$ as $\S_{\mathcal{N}_{k}(i)}$.

(1) Initialization: At the beginning of each frame, set every node to state $S$, that is, $s_{k}=S$ for all $k$. Then, we allow that every node $k$ generates a "contention request vector" $\mathbf{v}_{k}$ that randomly maps each neighboring node in list $\mathcal{N}_{k}$ to one of $M$ contention slots if node $k$ has traffic load intended to those neighbors. Here, we assume that $M$ is larger than the size of every neighbor list, that is, $M \geq\left|\mathcal{N}_{k}\right|$ for all $k$. The ratio of $M$ over $\left|\mathcal{N}_{k}\right|$ affects the probability of handshaking collisions. The larger is the ratio, the lower is the probability of handshaking collisions. We denote the $m$ th element of $\mathbf{v}_{k}$ as $v_{k}(m)$, which is

$$
v_{k}(m)= \begin{cases}j & \begin{array}{l}
\text { if node } k \text { has traffic to node } j, \text { and } j \\
\text { is mapped into contention slot } m,
\end{array} \\
0 \quad \text { otherwise. }\end{cases}
$$

In other words, the value of $v_{k}(m)$ is the index of the receiving node for which the transmitting node $k$ wants to contend during the contention slot $m$ for the upcoming data subframe. If $v_{k}(m)=0$, it means that, in the $m$ th contention slot, node $k$ will not contend for the upcoming data subframe.

(2) In contention slot $m$ : each node $k$ will first check its contention request vector. If $v_{k}(m)=0$, node $k$ eavesdrops ongoing handshaking within the neighborhood of range $\mathcal{R}$. (Naturally, we assume that the eavesdropping range $\mathcal{R}_{e}$ from each node is larger than the cooperative range $\mathcal{R}$. Furthermore, the carrier sense range $\mathcal{R}_{c}$, although not considered in this paper, would be even larger than $\mathcal{R}_{e}$.) If node $k$ hears any CTS or ACK packet, it retrieves the information from the packet and resets the states of the nodes in $\mathcal{N}_{k}$ accordingly. If $v_{k}(m)=j$ where $j>0$, node $k$ will try to 
finish the following three-way RTS-CTS-ACK handshaking with node $j$.

(i) RTS:

Node $k$ sends an RTS packet to node $j$ which contains the identity of node $k$, if the following conditions are satisfied:
(a) $s_{k}=S$;
(b) $s_{\mathcal{N}_{k}(i)} \neq R$ for all $i$.

(ii) CTS:

If node $j$ has successfully received the RTS packet from node $k$ and the following conditions are satisfied:
(a) $s_{j}=S$;
(b) $\mathcal{S}_{\mathcal{N}_{j}(i)}=S$ for all $i$;
(c) $v_{j}(m)=0$,

then node $j$ resets $\delta_{j}=R$ and $\delta_{\mathcal{N}_{j}\left(i_{k}\right)}=T$ where $i_{k}$ is the index for node $k$ in the table $\mathcal{N}_{j}$, and sends a CTS packet back to node $k$.

(iii) ACK:

If node $k$ has successfully received the CTS packet from node $j$, the node $k$ resets $\delta_{k}=T$ and $\delta_{\mathcal{N}_{k}\left(i_{j}\right)}=R$ where $i_{j}$ is the index of node $j$ in the table $\mathcal{N}_{k}$, and sends back the ACK packet.

During any contention slot, if there is a collision of control packets within the radius $\mathcal{R}$, the operation in that slot is abandoned. But the collision of control packets at a receiver due to transmitters outside the radius $\mathcal{R}$ is assumed to be resolvable by using coding with relatively high redundancy. If the ratio of $M$ over the number of nodes within the radius $\mathcal{R}$ is large, the probability of collision of control packets is small. As long as the control packets are much smaller than the data packets (i.e., the control subframe is much smaller than the data subframe), the network spectral efficiency is dominated by the throughput in the data subframe. This assumption will be our basis for throughput evaluation of D-SAM.

The idea of using control subframe for scheduling is not new, which can be traced back to the bit map concept as well as the work shown in [24]. But the study of the impact of the cooperative radius $\mathcal{R}$ on the network throughput is new and important for large-scale ad hoc networks.

Figure 4 illustrates a snapshot of the concurrent cochannel transmission pairs for a square network, which was determined by D-SAM for data transmission. The radius $\mathcal{R}=d_{a}$ was chosen, where $d_{a}$ is the spacing between two nearest neighbors. The number of contention slots was $M=$ 8. The full traffic loading condition, that is, $\zeta=1$, was assumed.

For D-SAM, we evaluate the network throughput in bitsmeter/s/Hz/node as follows:

$$
C_{\mathrm{D}-\mathrm{SAM}}=E\left\{\frac{1}{N} \sum_{n=1}^{N} d_{n} R_{\xi} s_{n}\right\}
$$

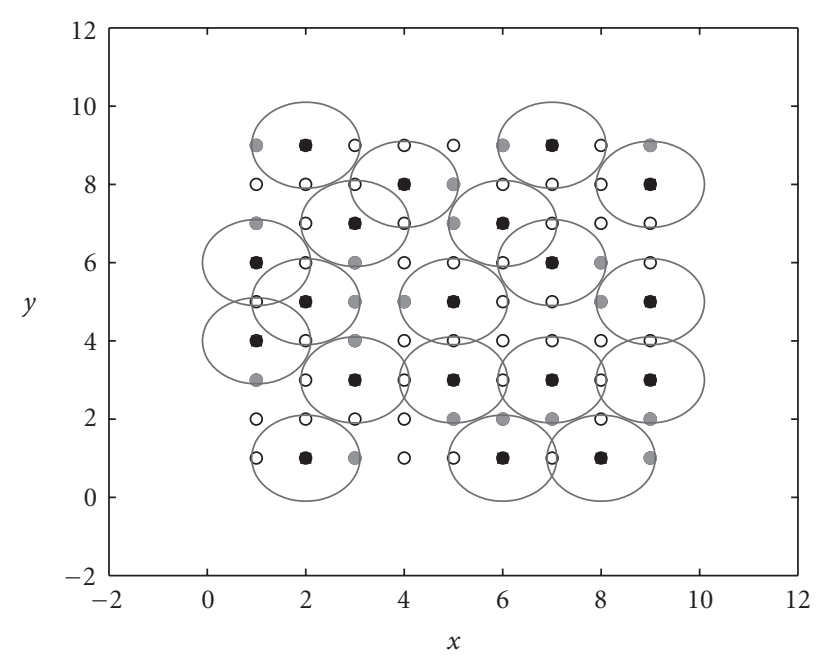

FIGURE 4: A snapshot of concurrent cochannel transmissions determined by the D-SAM protocol for a network in a regular square grid. $\mathcal{R}=d_{a}, M=8$, and $\zeta=1$ were used, where $d_{a}$ is the minimum distance between two adjacent nodes. The black nodes are the receiving nodes, and the grey nodes are the transmitting nodes.

where $E$ denotes expectation, $N$ is the total number of nodes in the network, $d_{n}$ is the distance between the $n$th receiving node and its transmitting node, $R_{\xi}$ is the packet spectral efficiency as defined before, $s_{n} \in\{0,1\}$, and $s_{n}=1$ if and only if a packet is intended for the $n$th node and the corresponding SINR is no less than $\xi$. In the simulation, the expectation is replaced by the average over many time frames. Each time frame also corresponds to an independent realization of Gaussian random channels. The distance weighting in (33) is different from the conversion formulas (from bits-hop/s/Hz/node to bits-meter/s/Hz/node) derived in [14] because the former does not take into account the fact that a typical multihop route between source node and destination node is not a straight line due to topology constraint. However, for regular topologies, the weighting used in (33) is slightly larger than that used in [14]. For an arbitrary topology, (33) represents an upper bound on the throughput in bits-meter/s/Hz/node.

\section{Loading Adaptive ALOHA}

Slotted ALOHA (or ALOHA for short) is a useful benchmark for throughput comparison. The protocol of ALOHA is as follows. In each time slot or frame, if a node $A$ has a packet to deliver to a neighboring node $B$, then the node $A$ transmits the packet to the node $B$ with a transmission probability $\varepsilon$. If the node $B$ is not transmitting in the same time slot, the node $B$ attempts to receive the packet from the node $A$.

The throughput of ALOHA can be shown as follows. Since each node has the probability $\zeta$ to have a packet for its neighbor, the effective probability for a node to choose to transmit is $\zeta \varepsilon$. Hence, the throughput of ALOHA in bitsmeter $/ \mathrm{s} / \mathrm{Hz} /$ node for networks of regular topologies is given 
by the following expression [14]:

$$
C_{\mathrm{ALOHA}}=\frac{\beta}{\sqrt{\rho}}(1-\zeta \varepsilon) \zeta \varepsilon R_{\xi} P_{d} .
$$

Here, as defined before, $R_{\xi}$ is the packet spectral efficiency, and $P_{d}=\operatorname{Pr}\{$ SINR $\geq \xi\}$ is the probability of packet detection. However, the statistics of SINR for ALOHA is different from that for SAM.

Note that the above expression (34) is for "per node" throughput, that is, in terms of bits-meter/s/Hz/node. (It is per every source node.) If there are total $N$ nodes where $N$ is large, the aggregated network throughput in bits-meter $/ \mathrm{s} / \mathrm{Hz}$ is $N$ times the expression (34). This expression has taken into account statistically all possible transmission patterns, which include the scenarios where multiple nodes are transmitting to a common receiver. When a node is in the receiving mode, it tries to decode the information from each of its neighboring nodes (say, nodes $A$ and $B$ ). When the receiving node tries to decode the information from node $A$, the signal from node $B$ (if any) will be treated as noise, and vice versa. A simple way to understand (34) is to first consider the network throughput in terms of bits-hop/s/Hz/node [2], which corresponds to the (averaged) throughput for each pair of neighboring nodes. The probability that each pair of neighboring nodes forms a transceiver is given by $(1-\zeta \varepsilon) \zeta \varepsilon$. Given that a pair of neighboring nodes forms a transceiver, the amount of information transferred between them is $R_{\xi} P_{d}$. Hence, $(1-\zeta \varepsilon) \zeta \varepsilon R_{\xi} P_{d}$ is the throughput in bitshop $/ \mathrm{s} / \mathrm{Hz} /$ node. The factor $\beta / \sqrt{\rho}$ converts the throughput from bits-hop/s/Hz/node to bits-meter/s/Hz/node, see [14]. Also note that the expression (34) assumes one type of multiuser detection at each receiving node. However, the packets from multiple transmitters are not jointly encoded.

For throughput analysis of ALOHA, we will only consider SISO channels. Then, given that a node transmits a packet and one of its neighboring nodes receives the packet, the signal received by the receiving node can be modeled as

$$
y_{0}=h_{0} x_{0}+\sum_{j>0} h_{j} x_{j} u_{j}+w_{0}
$$

where $x_{0}$ is the desired signal, $h_{0}$ is the channel coefficient between the desired transmitter-receiver pair, $x_{j}$ for $j>0$ is the interfering signal from node $j, h_{j}$ for $j>0$ is the channel coefficient between the interfering node $j$ and the receiving node, and $w_{0}$ is the noise. We assume Gaussian fading channels and Gaussian noise. Here, $u_{j} \in\{0,1\}$ are i.i.d. binary random variables with $\operatorname{Pr}\left\{u_{j}=1\right\}=\zeta \varepsilon$. Then, the instantaneous SINR in $y_{0}$ in each time slot is

$$
\operatorname{SINR}=\frac{g_{0} P}{\sigma^{2}+\sum_{j>0} g_{j} u_{j} P},
$$

where $P$ is the transmitted power from each transmitting node, $\sigma^{2}$ is the noise variance, $g_{i}=\left\|h_{i}\right\|^{2}$ is an exponentially distributed random variable with the mean $\mu_{i}=d_{i}^{-\alpha}$, and $d_{i}$ is the distance between the node $i$ and the receiver.
Unlike (5), we now have

$$
\begin{aligned}
P_{d} & =\operatorname{Pr}\{\operatorname{SINR} \geq \xi\} \\
& =\operatorname{Pr}\left\{\frac{g_{0}}{\sigma^{2} / P+\sum_{j>0} g_{j} u_{j}} \geq \xi\right\} \\
& =E_{\left\{g_{i}, u_{i}, i>0\right\}}\left[\int_{\left(\sigma^{2} / P+\sum_{j>0} g_{j} u_{j}\right) \xi}^{\infty} \frac{1}{\mu_{0}} e^{-x / \mu_{0}} d x\right] \\
& =E_{\left\{g_{i}, u_{i}, i>0\right\}} \exp \left\{-\frac{\left(\sigma^{2} / P+\sum_{j>0} g_{j} u_{j}\right) \xi}{\mu_{0}}\right\} \\
& =\exp \left\{-\frac{\sigma^{2} \xi}{P \mu_{0}}\right\} \prod_{j>0} E_{g_{i}, u_{i}}\left\{\exp \left(-\frac{g_{i} u_{i} \xi}{\mu_{0}}\right)\right\} \\
& =\exp \left\{-\frac{\sigma^{2} \xi}{P \mu_{0}}\right\} \prod_{j>0} E_{u_{i}}\left\{\frac{1}{1+u_{i} \mu_{i} \xi / \mu_{0}}\right\} \\
& =\exp \left\{-\frac{\sigma^{2} \xi}{P \mu_{0}}\right\} \prod_{j>0}\left[\zeta \varepsilon \frac{1}{\xi \mu_{j} / \mu_{0}+1}+(1-\zeta \varepsilon)\right] .
\end{aligned}
$$

The above analysis is similar to one in [13]. Since $\zeta$ and $\varepsilon$ always appear in the product form $\zeta \varepsilon$, given that all other parameters are fixed, there is an optimal choice for the product, which is to be denoted by $p^{*}$. Assuming that each node knows the traffic loading condition as measured by $\zeta$, then a loading adaptive ALOHA should adopt the following transmission probability:

$$
\varepsilon= \begin{cases}1 & \zeta \leq p^{*}, \\ \frac{p^{*}}{\zeta} & \zeta>p^{*} .\end{cases}
$$

For the throughput comparison shown next, we will use the loading adaptive ALOHA.

\section{Throughput Evaluation}

In this section, we will illustrate and compare the throughput of O-SAM, D-SAM, and ALOHA. We will use the following list of assumptions. All network topologies to be considered have the unit node density $\rho=1$. All channel coefficients are independent realizations of complex Gaussian random variables from frame to frame. We choose the path loss exponent $\alpha=4$ unless specified otherwise. By SIMO, we mean $1 \times 4$ SIMO, and by MIMO, we mean $4 \times 4$ MIMO. For O-SAM, we will consider a large network of 245 nodes on three regular grids as shown in Figure 2. The subnet partitions shown in this figure are already optimized for OSAM under the full load condition. For the Fourier series expansion (12) and (13), we choose $K=500$ and $T=$ 50. These values were confirmed to be sufficiently large. For D-SAM, we will consider the three regular topologies as well as 20 random topologies. Each random topology consists of 300 nodes positioned by the two-dimensional Poisson random process. We will use $M=60$ with which the probability of control packet collision is negligible as observed in simulations. For ALOHA, we will only consider the square topology and SISO channels. 


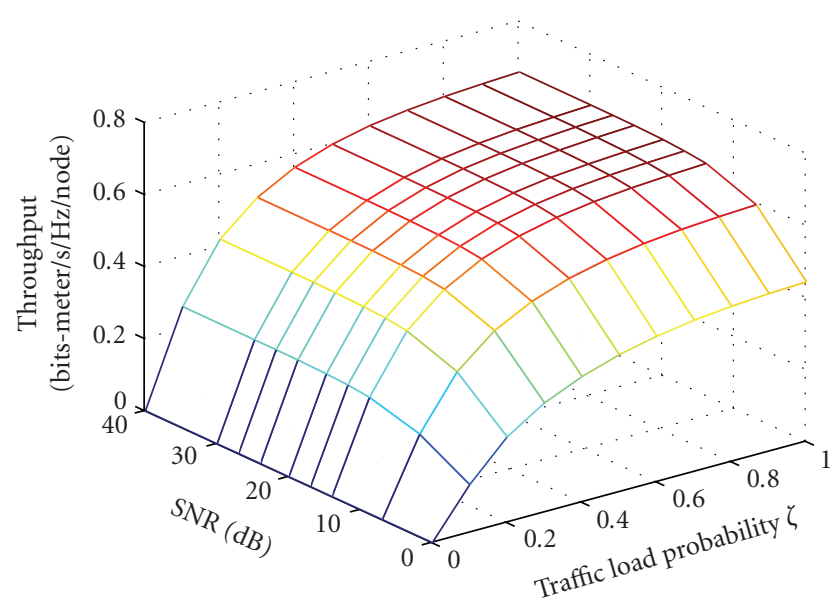

FIgURE 5: Throughput of O-SAM versus load probability $\zeta$ and SNR. We used $\rho=1$, square topology, and SISO channels.

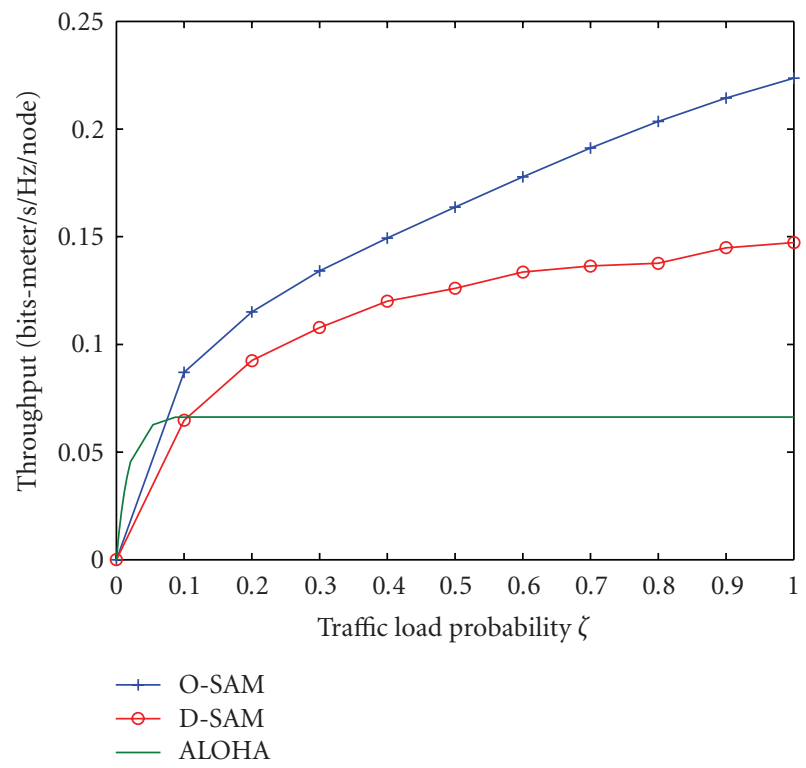

FIgUre 6: Throughput comparison of O-SAM, D-SAM, and ALOHA. We used $\rho=1, \mathrm{SNR}=40 \mathrm{~dB}$, square topology, and SISO channels.

Figure 5 shows the throughput of O-SAM versus SNR and the traffic load probability $\zeta$. For each pair of SNR and $\zeta$, the throughput was maximized over $\xi$ (the target SINR) and $\theta$ (the channel gain threshold). The square topology as shown in Figure 2 was used. The Gaussian SISO channels were considered. This figure is to highlight the fact that the network throughput is saturated to a constant when SNR is large. In the sequel, we will choose SNR $=10 \log _{10}\left(P / \sigma^{2}\right)=$ $40 \mathrm{~dB}$ unless otherwise specified.

Figure 6 compares the throughput of O-SAM, D-SAM, and ALOHA versus the traffic load probability $\zeta$. For each $\zeta$, the throughput of O-SAM was maximized over both $\xi$ and $\theta$, and the throughput of D-SAM was maximized over $\xi$ and $\mathcal{R}$ (the cooperative range). The square topology as shown in Figure 2 and the Gaussian SISO channels were considered.

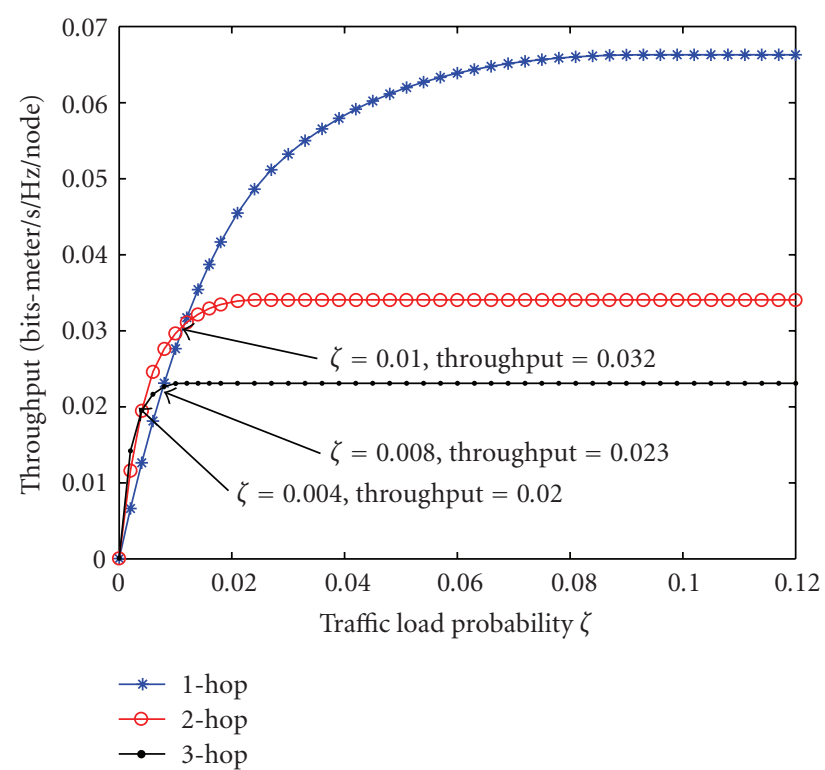

FIgURE 7: Throughput of ALOHA with different transmission ranges: 1-hop, 2-hop, and 3-hop ranges. We used $\rho=1$, square topology, and SISO channels. The transmission power for each of the three cases is adjusted so that the SNR (excluding interference) at every receiver is $40 \mathrm{~dB}$.

We see that as long as $\zeta>10 \%$, both O-SAM and D-SAM yield higher throughput than ALOHA. In other words, only when the traffic load is low, does ALOHA yield a higher throughput. Also note that the transmission probability of ALOHA is optimized for each $\zeta$. But the spacing for O-SAM and the cooperative radius for D-SAM are optimized only for $\zeta=1$. If those parameters are optimized for each $\zeta$, the throughput curves for O-SAM and D-SAM would be higher for $\zeta<1$. As expected, the throughput of D-SAM is lower than that of O-SAM. This is because the concurrent cochannel transmissions for D-SAM are not as ideal as those for O-SAM. This figure shows that the throughput of DSAM is about two thirds of that of O-SAM in the full load condition.

Figure 7 illustrates the throughput of ALOHA for 1-hop, 2-hop, and 3-hop distance transmissions. Note that bitsmeter $/ \mathrm{s} / \mathrm{Hz} /$ node is a distance-weighted throughput unit. By 2-hop distance transmission, for example, we mean that the transmission distance between the transmitter and the receiver equals two times the shortest distance between two adjacent nodes. For each of the three cases, we adjusted the transmission power $P$ such that the SNR of the received signal is kept at $40 \mathrm{~dB}$. This means that the transmission power used for 2-hop distance transmission is $2^{\alpha}$ times higher than that for 1-hop distance transmission, and the transmission power used for 3-hop distance transmission is $3^{\alpha}$ times higher than that for 1-hop distance transmission. The same square topology as shown in Figure 2 and the Gaussian fading SISO channels were considered. For each $\zeta$, the throughput was maximized over $\xi$. We see that only when the traffic load is very low (that is, $\zeta<1 \%$ ), is the throughput of 2-hop distance transmission better than that 


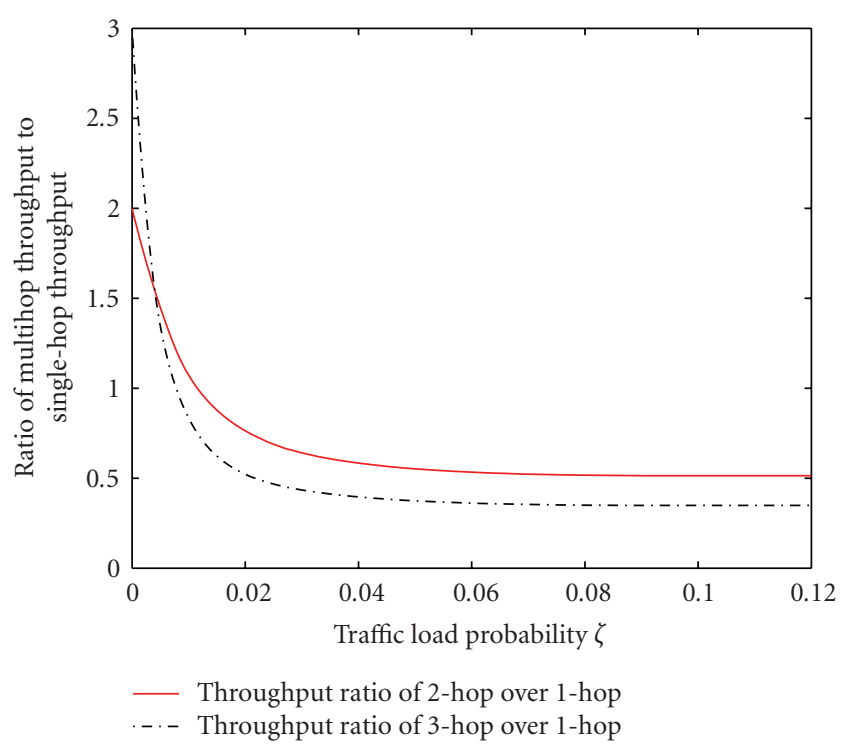

FIGURE 8: Ratios of throughput: 2-hop range over 1-hop range, and 3-hop rang over 1-hop range. All conditions are the same as for Figure 7.

of 1-hop distance transmission. In order for 3-hop distance transmission to be better than 2-hop distance transmission, the traffic load probability $\zeta$ needs to be less than $0.4 \%$.

In Figure 8, we show the ratio of the "2-hop distance" throughput over the "1-hop distance" throughput and the ratio of the "3-hop distance" throughput over the "1-hop distance" throughput. These ratios are lower than one unless the traffic load probability $\zeta$ is very small. When $\zeta$ approaches zero, the two ratios become two and three, respectively.

Figures 7 and 8 suggest that for peer-to-peer networks, the shortest distance transmission is the most efficient in both spectrum and energy unless the traffic load is extremely low.

Figure 9 compares the throughput of O-SAM and DSAM for each of SISO, SIMO, and MIMO cases. For O-SAM, the throughput was maximized over $\xi$ and $\theta$. For D-SAM, the throughput was maximized over $\xi$ and $\mathcal{R}$. The square network was considered. This figure illustrates that multiple antennas can significantly improve the network throughput.

Figure 10 compares the throughput of O-SAM and DSAM for each of the three topologies: square, triangle, and hexagon. A useful observation is that O-SAM with the hexagonal network has a much higher throughput than all other situations. It is also useful to note here that the optimal subnet partition of the hexagonal network for O-SAM as shown in (2) is identical to that for C-SAM as shown in [14]. Hence, for the hexagonal topology, the throughput gain due to the opportunistic transmitter selection is not compromised by any change of subnet partition. This is not the case for the other two topologies. Although the throughput of D-SAM is not as high as that of O-SAM, DSAM works with any topology.

Figure 11 illustrates the $\xi$-optimized throughput of DSAM versus the cooperative range $\mathcal{R}$. It is interesting to

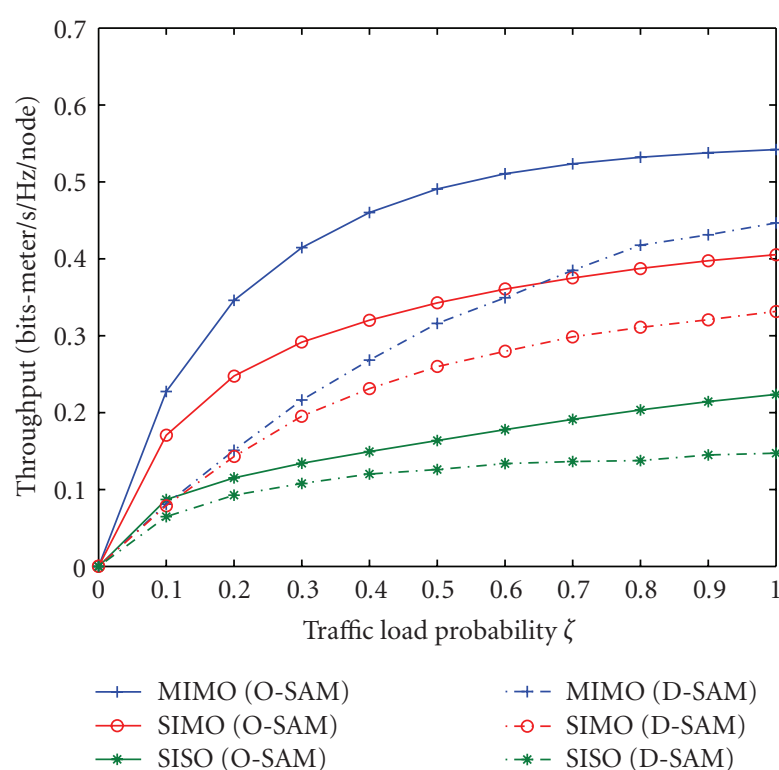

FIgure 9: Throughput of O-SAM and D-SAM for SISO, SIMO, and MIMO channels. We used $\rho=1$, SNR $=40 \mathrm{~dB}$, and square topology.

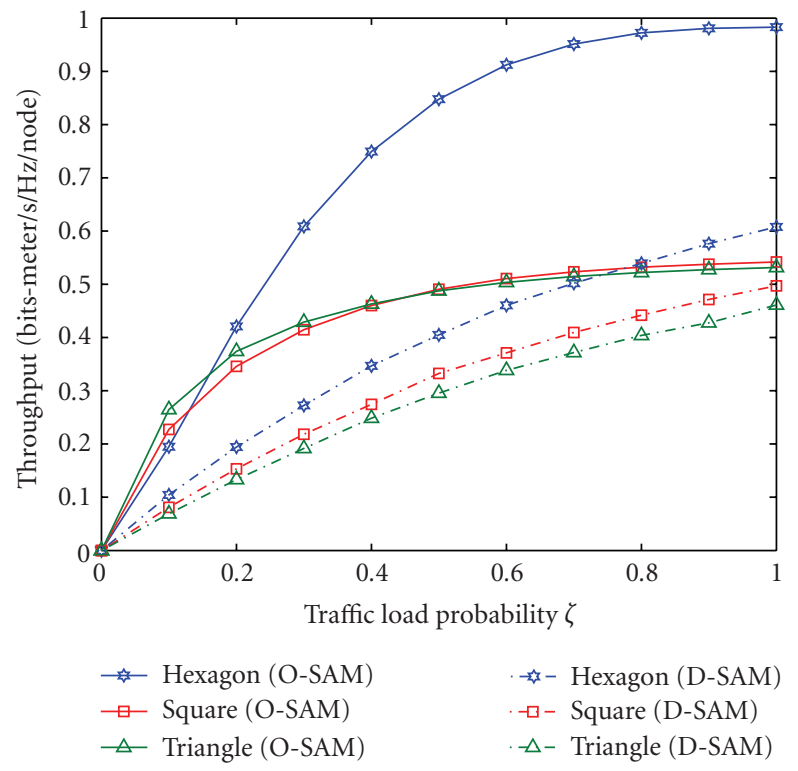

Figure 10: Throughput of O-SAM and D-SAM for different network topologies. We used $\rho=1, \mathrm{SNR}=40 \mathrm{~dB}$, and $4 \times 4 \mathrm{MIMO}$ channels.

observe that for all three regular topologies, the optimal cooperative range $\mathcal{R}^{*}$ satisfies $d_{a} \leq \mathcal{R}^{*}<d_{b}$. Here, $d_{a}$ is the shortest distance between two adjacent nodes, and $d_{b}$ is the shortest distance between two nodes that are two hops apart. Clearly, when $\mathcal{R}<d_{a}$, the throughput for the regular topologies should be zero. We also see that for the regular topologies, the throughput in the interval $d_{a} \leq \mathcal{R}<d_{b}$ is essentially constant where the variations due to random subnet partitions and random channel realizations are small 


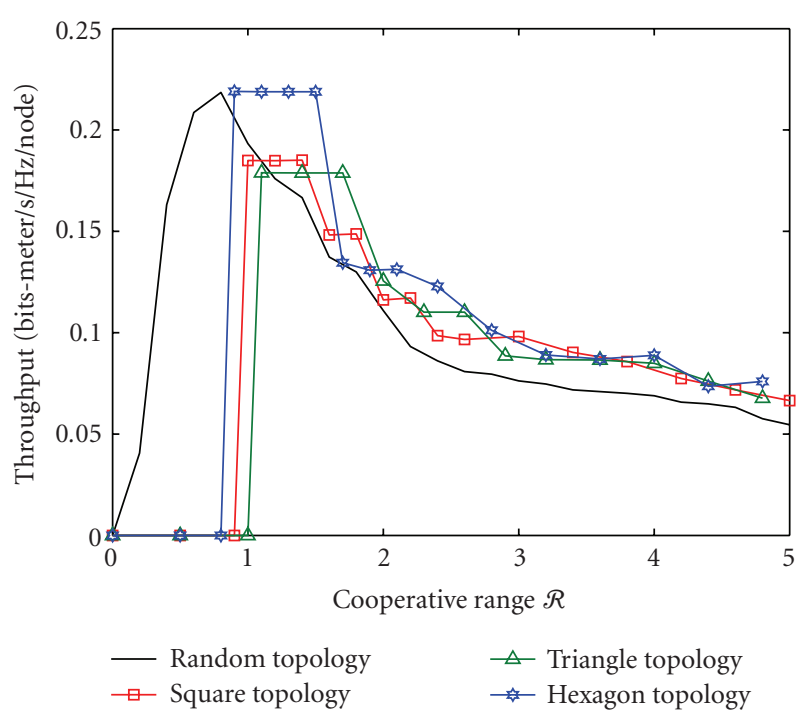

FIGURE 11: Throughput of D-SAM versus the cooperative range $\mathcal{R}$. We used $\rho=1, \zeta=1$, SNR $=40 \mathrm{~dB}$, and SISO channels.

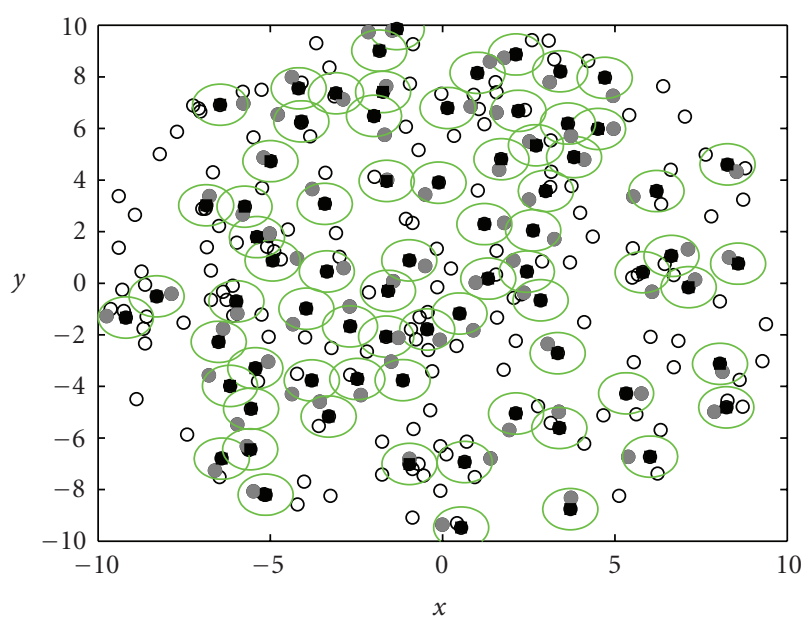

FIGURE 12: A snapshot of subnet partition of a random network by D-SAM with $\mathcal{R}=0.8$. The black nodes are the receivers, and the grey nodes are the transmitters. We used $\rho=1$ and $\zeta=1$. Each circle shown has the radius 0.8 .

and not perceivable from this figure. Under $d_{a} \leq \mathcal{R}<d_{b}$, the corresponding optimal target SINR is $\xi^{*} \approx 4$.

Given $\rho=1$, we have $d_{a}=1$ and $d_{b}=\sqrt{2} d_{a}=1.41$ for square; $d_{a}=\sqrt{2 / \sqrt{3}}=1.07$ and $d_{b}=\sqrt{3} d_{a}=1.85$ for triangle; and $d_{a}=\sqrt{4 /(3 \sqrt{3})}=0.877$ and $d_{b}=\sqrt{3} d_{a}=1.52$ for hexagonal [14]. We will restrict $d_{a}$ and $d_{b}$ to be defined as above only for the regular topologies.

As observed in our simulation, this optimal condition $d_{a} \leq \mathcal{R}^{*}<d_{b}$ also holds for $\alpha=3$. This observation interestingly supports the two-hop rule adopted in MSHDSCH of IEEE 802.16. But the corresponding $\xi^{*}$ decreases as the path loss exponent $\alpha$ decreases. We found that $\xi^{*}$ is somewhere between 1.5 and 2 when $\alpha=3$. Note that the spectral efficiency of each packet is governed by the value of $\xi$, that is, $R_{\xi}=\log _{2}(1+\xi)$.

Figure 11 also shows that for $\mathcal{R}<d_{a}$, the throughput of the random topologies is nonzero, and furthermore it peaks at $\mathcal{R}=0.8$. It is important to note that the throughput under $\mathcal{R}<d_{a}$ is not very meaningful. This is because when $\mathcal{R}<d_{a}$, the distance between many adjacent nodes is larger than $\mathcal{R}$ so that there is no direct link between them. In fact, under $\mathcal{R}=0.8$, many nodes are not even connected with others, which is illustrated in Figure 12. In such a case, the expression defined in (33) is only a very loose upper bound on the network throughput.

The detailed insights from each simulation example have been presented above. Our overall observations are summarized in the next section.

\section{Conclusion}

We have presented a further development of synchronous array method (SAM) as a medium access control scheme for stationary ad hoc wireless networks. We have focused on intranetwork throughput enhancement for a large network where any node can be a source node or a destination node. We have used the distance-weighted throughput measure: bits-meter/s/Hz/node. We have presented and evaluated two SAM-based schemes: O-SAM and D-SAM. These two schemes require different levels of centralization and cooperation within the network.

With O-SAM, the subnet partition within each time frame needs to be predetermined. Provided that the channel coherence time is sufficiently long, local channel estimation is feasible which allows opportunistic exploitation of channel gains within each subnet. The exchange of local information (other than large data packets) can be done via ALOHAbased protocols. In order to induce variations of channel gains, multiple antennas (and a transmit beam vector randomly selected for each frame) can be used at each node. The throughput of O-SAM has been shown to be much higher than that of D-SAM.

With D-SAM, the subnet partition within each time frame is decided by the network locally and dynamically as governed by the cooperative radius (which is smaller than the eavesdropping radius and the carrier sense radius). For networks of sufficiently long channel coherence time, the spectral overhead for exchanges of control packets can be affordable or even negligible compared to the exchanges of data packets. In this case, the network throughput is primarily affected by the subnet partition in each time frame. The cooperative radius $\mathcal{R}$ has a major effect on the size of each subnet and hence the network throughput. For networks of regular topologies and full traffic load, the optimal value of $\mathcal{R}$ has been shown to be anywhere between $d_{a}$ and $d_{b}$ where $d_{a}$ is the shortest distance between two adjacent nodes and $d_{b}$ is the shortest distance between two nodes that are two hops apart. This result interestingly supports the two-hop rule adopted in MSH-DSCH in IEEE 802.16.

We have also compared the throughput of O-SAM and D-SAM with the throughput of ALOHA under a varying 
probability $\zeta$ of traffic load. It has been shown that ALOHA yields lower throughput than O-SAM and D-SAM unless $\zeta$ is small, for example, less than $10 \%$. We have further examined the effect of the distance of each transmission on the distance-weighted throughput. We have found that the shortest distance transmission leads to the highest throughput unless $\zeta$ is very small, for example, less than $1 \%$.

\section{Acknowledgments}

This work was supported in part by the U.S. Army Research Office under the MURI Grant no. W911NF-04-1-0224, the U.S. Army Research Laboratory under the Collaborative Technology Alliance Program, and the U.S. National Science Foundation under Grant no. TF-0514736.

\section{References}

[1] P. Gupta and P. R. Kumar, "The capacity of wireless networks," IEEE Transactions on Information Theory, vol. 46, no. 2, pp. 388-404, 2000.

[2] Y. Hua, Y. Huang, and J. J. Garcia-Luna-Aceves, "Maximizing the throughput of large ad hoc wireless networks," IEEE Signal Processing Magazine, vol. 23, no. 5, pp. 84-94, 2006.

[3] U. C. Kozat and L. Tassiulas, "Throughput capacity of random ad hoc networks with infrastructure support," in Proceedings of the 9th Annual International Conference on Mobile Computing and Networking (MobiCom '03), pp. 55-65, San Diego, Calif, USA, September 2003.

[4] B. Liu, Z. Liu, and D. Towsley, "On the capacity of hybrid wireless networks," in Proceedings of the 22nd Annual Joint Conference on IEEE Computer and Communications Societies (INFOCOM '03), vol. 2, pp. 1543-1552, San Francisco, Calif, USA, March-April 2003.

[5] A. Agarwal and P. R. Kumar, "Capacity bounds for ad hoc and hybrid wireless networks," ACM SIGCOMM Computer Communication Review, vol. 34, no. 3, pp. 71-81, 2004.

[6] S. Toumpis, "Capacity bounds for three classes of wireless networks: asymmetric, cluster, and hybrid," in Proceedings of the 5th ACM International Symposium on Mobile Ad Hoc Networking and Computing (MobiHoc '04), pp. 133-144, Tokyo, Japan, May 2004.

[7] S. Yi, Y. Pei, and S. Kalyanaraman, "On the capacity improvement of ad hoc wireless networks using directional antennas," in Proceedings of the 4th ACM International Symposium on Mobile Ad Hoc Networking and Computing (MobiHoc '03), pp. 108-116, Annapolis, Md, USA, June 2003.

[8] R. Negi and A. Rajeswaran, "Capacity of power constrained ad-hoc networks," in Proceedings of the 23rd Annual Joint Conference of IEEE Computer and Communications Societies (INFOCOM '04), vol. 1, pp. 443-453, Hong Kong, March 2004.

[9] X. Tang and Y. Hua, "Capacity of ultra-wideband powerconstrained ad hoc networks," IEEE Transactions on Information Theory, vol. 54, no. 2, pp. 916-920, 2008.

[10] O. Levegue, A. Ozgur, and D. Tse, "How does the information capactiy of ad hoc networks scale?" in Proceedings of the 44th Annual Allerton Conference on Communication, Control and Computing, Allerton Park, Ill, USA, September 2006.

[11] J. L. Massey, Ed., "Special Issue on Random-Access Communications," IEEE Transactions on Information Theory, vol. 31, no. 2, 1985 .
[12] "IEEE standard for wireless LAN medium access control (MAC) and physical layer (PHY) specifications," November 1997.

[13] X. Liu and M. Haenggi, "Throughput analysis of fading sensor networks with regular and random topologies," EURASIP Journal on Wireless Communications and Networking, vol. 2005, no. 4, pp. 554-564, 2005.

[14] K. Hong and Y. Hua, "Throughput analysis of large wireless networks with regular topologies," EURASIP Journal on Wireless Communications and Networking, vol. 2007, Article ID 26760, 11 pages, 2007.

[15] M. Vutukuru, K. Jamieson, and H. Balakrishnan, "Harnessing exposed terminals in wireless networks," in Proceedings of the 5th USENIX Symposium on Networked Systems Design and Implementation, pp. 59-72, San Francisco, Calif, USA, April 2008.

[16] B. Zhao and Y. Hua, "A distributed medium access control scheme for a large network of wireless routers," IEEE Transactions on Wireless Communications, vol. 7, no. 5, pp. 1614-1622, 2008.

[17] S. Adireddy and L. Tong, "Exploiting decentralized channel state information for random access," IEEE Transactions on Information Theory, vol. 51, no. 2, pp. 537-561, 2005.

[18] B. Zhao and Y. Hua, "Distributed medium access for a large wireless mesh network with multiple antenna elements on each node," in Proceedings of IEEE International Conference on Acoustics, Speech and Signal Processing (ICASSP '07), vol. 3, pp. 589-592, Honolulu, Hawaii, USA, April 2007.

[19] "IEEE standard for local and metropolitan area networks IEEE 802.16," October 2004.

[20] M. Cao, W. Ma, Q. Zhang, and X. Wang, "Analysis of IEEE 802.16 mesh mode scheduler performance," IEEE Transactions on Wireless Communications, vol. 6, no. 4, pp. 1455-1464, 2007.

[21] K. Hong, Y. Hua, and A. Swami, "Distributed and cooperative link scheduling for large-scale multi-hop wireless networks," EURASIP Journal on Wireless Communications and Networking, vol. 2007, Article ID 34716, 9 pages, 2007.

[22] A. Shah and A. M. Haimovich, "Performance analysis of maximal ratio combining and comparison with optimum combining for mobile radio communications with cochannel interference," IEEE Transactions on Vehicular Technology, vol. 49, no. 4, pp. 1454-1463, 2000.

[23] M. Kang and M.-S. Alouini, "Largest eigenvalue of complex wishart matrices and performance analysis of MIMO MRC systems," IEEE Journal on Selected Areas in Communications, vol. 21, no. 3, pp. 418-426, 2003.

[24] T. J. Shepard, Decentralized channel management in scalable multihop spread-spectrum packet radio networks, Ph.D. dissertation, Massachusetts Institute of Technology, Cambridge, Mass, USA, 1995. 